\title{
Strengths and Difficulties Questionnaire scores and mental health in looked after children
}

\author{
Anna Goodman and Robert Goodman
}

\begin{abstract}
Summary
British local authorities are required to monitor the mental health of looked after children using mean Strengths and Difficulties Questionnaire (SDQ) scores from parents or carers. This assumes that differences in mean SDQ scores reflect genuine differences in child mental health in this group, something we examined using nationally representative surveys ( $n=1391$, age $5-16)$. We found that the SDQ was a genuinely dimensional measure of mental
\end{abstract}

health in these children and provided accurate estimates of disorder prevalence.

\section{Declaration of interest}

A.G. and R.G. are directors and part-owners of Youthinmind, which provides no-cost and low-cost software and websites related to the SDQ and the Development and Well-Being Assessment.
The mental health of children looked after by the state (e.g. in residential or foster family care) is a priority for both policymakers and practitioners. ${ }^{1}$ Since 2009 , the British government has therefore required all local authorities to administer the Strengths and Difficulties Questionnaire (SDQ) ${ }^{2}$ annually to the primary carers of all children aged 4-16 who have been looked after for at least 1 year. $^{3}$ Local authorities are then required to report the mean score to the Department for Education, with the intention that trends can be monitored over time and/or comparisons made between comparable local authorities.

The parent SDQ is a good screening device for mental disorders in looked after children ${ }^{4,5}$ with 'high' SDQ scores predicting much greater rates of disorder than 'low' scores. If comparisons across local authorities or over time are to be valid, however, more than this is required: it needs to be demonstrated that any difference in mean SDQ scores corresponds (on average) to genuine differences in mental health. This appears to be true in the general British population. In individual-level analyses, each one-point increase in mean symptom SDQ score predicts a higher prevalence of disorder. ${ }^{6}$ Likewise in population-level analyses, mean SDQ scores predict the prevalence of disorder in an accurate and unbiased manner across subpopulations defined by multiple child, family and area characteristics (such as ethnicity, family type, area deprivation). ${ }^{7}$ This allowed us to derive and validate UK 'SDQ prevalence estimators'.

Yet, although encouraging, these general population findings cannot be assumed to apply to looked after children. First, nationally representative British surveys of looked after children report a disorder prevalence of $45 \%,{ }^{1}$ which is much higher than the prevalence in the general population $(9.4 \%$ overall prevalence) or in any of the subpopulations we studied (median 9.8\%, interquartile range $7.3-14.5 \%$ ). Second, the prevalence estimators were derived using data mostly provided by biological parents. By contrast, informants for looked after children are usually foster parents or care workers who may respond differently. This report therefore uses a nationally representative sample to examine whether, among looked after children (a) the parent SDQ is a genuinely dimensional measure of child mental health, and (b) the parent SDQ prevalence estimator equation is accurate.

\section{Method}

\section{Sample}

We combined data from three nationally representative surveys (one each in England, Scotland and Wales) of looked after children aged 5-17. ${ }^{1}$ In 2002-2003, random samples of looked after children were selected from the relevant databases in each country, and primary carers, teachers and youth over 11 were asked to complete the SDQ and the Development and Well-Being Assessment (DAWBA). ${ }^{8}$ In total, 1391 looked after children (595 females) aged 5-16 provided DAWBA and parent SDQ data (57\% participation rate; we excluded 127 children aged over 16 to match the age range monitored by local authorities). We divided these children by placement type: living with natural parents $(n=190)$; living with kin $(n=165)$; living with unrelated foster parents $(n=781)$; or living in residential care, i.e. non-family placements $(n=255)$.

For comparison, we present data from the 1999 and 2004 British Child and Adolescent Mental Health surveys, two nationally representative surveys of children aged 5-16 in private households. $^{7}$ In total, 18205 children (8967 females) provided DAWBA and parent SDQ data (69\% participation rate). These were divided into fifths according to small-area deprivation. ${ }^{9}$

\section{Measures}

The Strengths and Difficulties Questionnaire (SDQ) is a measure of mental health problems in children aged 4-17 that can be administered to parents/carers, teachers and children aged 11 or over. $^{2}$ Its 20 items relating to emotional symptoms, conduct problems, hyperactivity and peer problems are summed to create a 'total difficulty' symptom score ranging from 0 to 40 . We have previously developed and validated UK 'SDQ prevalence estimators' that convert a subpopulation's mean symptom score into an estimated prevalence of disorder, adjusting for the subpopulation's age and gender composition ${ }^{7}$ (see the online supplement for the prevalence estimator equations, and a link to online look-up tables).

The DAWBA is a detailed psychiatric interview for parents/ carers and youth, and a briefer questionnaire for teachers. It includes a fully structured section followed by open-ended descriptions by respondents of problem areas. Experienced clinicians then assign diagnoses according to DSM-IV, ${ }^{10}$ using both the closed and open DAWBA responses, and triangulating information across informants.

\section{Analysis}

Our individual-level analysis involved taking looked after children with any given SDQ score and plotting this against the measured prevalence of disorder for that SDQ score (disorder defined as 
(a)

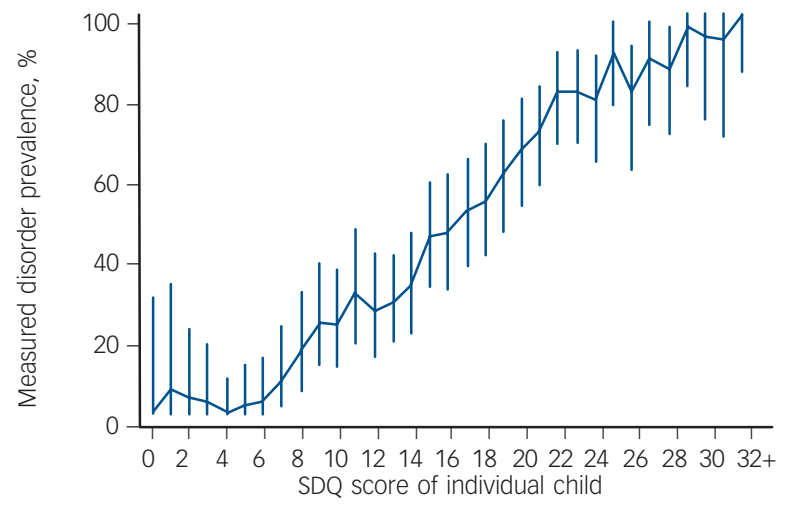

(b)

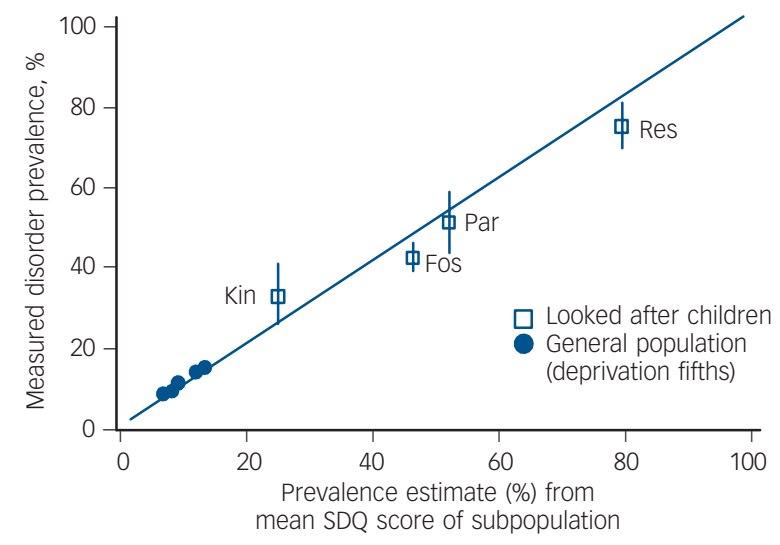

Fig. 1 Using mean parent Strengths and Difficulties Questionnaire (SDQ) scores to predict the percentage prevalence (95\% Cl) of child mental health disorder among looked after children: performance at the (a) individual and (b) population level.

Par, living with natural parents; Kin, living with kin; Fos, living with foster parents; Res, residential care. See online Table DS1 for tabulated population-level data. In individual-level analysis, SDQ points were grouped once the number of individuals fell to below 10.

receiving at least one DSM-IV DAWBA diagnosis). Our population-level analysis involved calculating the mean parent SDQ score for four subpopulations of looked after children (defined by placement type) and using the SDQ prevalence estimators to estimate disorder prevalence. We then plotted these estimated prevalences against the measured prevalence of disorder in that subpopulation, presenting general population data for comparison. All analyses used Stata12 for Windows 2007.

\section{Results}

Each one-point increase in SDQ score among looked after children generally corresponded to an increased prevalence of clinical disorder (Fig. 1(a)). The only possible exception was for very low scores $(0-4)$; given that such low scores were rare in this sample this could reflect regression to the mean or simply the small sample sizes at this low end. The parent SDQ prevalence estimator also performed well in our population-level analysis (Fig. 1(b)). Subpopulations of looked after children with higher mean SDQ scores also had a higher prevalence of disorder, and the SDQ prevalence estimators provided good approximate estimates of these (discrepancies 3-7\% across our four subpopulations, in the context of measured prevalences of $31-73 \%$ ).

\section{Discussion}

Our findings demonstrate that the parent SDQ provides a genuinely dimensional measure of mental health in looked after children, despite their having a much higher prevalence of disorder than the general population. Thus, any difference between groups of looked after children in their mean SDQ score will, on average, reflect real differences in their mental health. This supports the use of mean SDQ scores to compare local authorities and monitor trends over time. Moreover, mean parent SDQ scores also generated good estimates of disorder prevalence using the SDQ prevalence estimators. ${ }^{7}$ This may be useful when seeking to translate findings for local policy-makers and practitioners, as one can turn non-intuitive mean scores into intuitive caseness rates.

Our findings therefore support the British government's current use of mean parent SDQ symptom scores to monitor the mental health of looked after children. Given that the parent SDQ is also a valid screening measure for psychiatric disorder in looked after children ${ }^{4,5}$ the parent SDQs collected could additionally be used to identify high-risk individuals who warrant more detailed assessment. We therefore hope the recent requirement placed on local authorities to administer the parent SDQ to looked after children will permit monitoring and screening programmes that can inform both public health and clinical interventions. Beyond Britain, the SDQ has been validated for screening looked after children in Canada, ${ }^{4}$ and other countries may also wish to examine its utility.

Anna Goodman, MSC, PhD, Faculty of Epidemiology and Population Health, London School of Hygiene and Tropical Medicine, London; Robert Goodman, FRCPsych, $\mathrm{PhD}$, Institute of Psychiatry, King's College London, UK

Correspondence: Anna Goodman, Faculty of Epidemiology and Population Health, London School of Hygiene and Tropical Medicine, Keppel Street, London WC1E 7HT, UK. Email: anna.goodman@Ishtm.ac.uk

First received 16 Oct 2011, final revision 20 Dec 2011, accepted 13 Feb 2012

\section{References}

1 Ford T, Vostanis P, Meltzer H, Goodman R. Psychiatric disorder among British children looked after by local authorities: comparison with children living in private households. Br J Psychiatry 2007; 190: 319-25.

2 Goodman R. Psychometric properties of the strengths and difficulties questionnaire. J Am Acad Child Adolesc Psychiatry 2001; 40: 1337-45.

3 Audit Commission. NI 58 Emotional and Behavioural Health of Looked After Children. Audit Commission, 2011 (http://www.audit-commission.gov.uk/ performance-information/performance-data-collections-and-guidance/nis/ Pages/NI058emotionalandbehaviouralhealthoflookedafterchildren.aspx).

4 Marquis RA, Flynn RJ. The SDQ as a mental health measurement tool in a Canadian sample of looked-after young people. Vulnerable Child Youth Stud 2009; 4: 114-21.

5 Goodman R, Ford T, Corbin T, Meltzer H. Using the Strengths and Difficulties Questionnaire (SDQ) multi-informant algorithm to screen looked-after children for psychiatric disorders. Eur Child Adolesc Psychiatry 2004; 13 (suppl 2): II25-31.

6 Goodman A, Goodman R. Strengths and difficulties questionnaire as a dimensional measure of child mental health. J Am Acad Child Adolesc Psychiatry 2009; 48: 400-3.

7 Goodman A, Goodman R. Population mean scores predict child mental disorder rates: validating SDQ prevalence estimators in Britain. J Child Psychol Psychiatry 2011; 52: 100-8.

8 Goodman R, Ford T, Richards H, Gatward R, Meltzer H. The Development and Well-Being Assessment: description and initial validation of an integrated assessment of child and adolescent psychopathology. J Child Psychol Psychiatry 2000; 41: 645-55.

9 Office of the Deputy Prime Minister. The English Indices of Deprivation 2004 (Revised). Office of the Deputy Prime Minister, 2004 (http:// www.communities.gov.uk/documents/communities/pdf/131209.pdf).

10 American Psychiatric Association. Diagnostic and Statistical Manual of Mental Disorders (4th edn) (DSM-IV). APA, 1994. 